\title{
Bloch Wave Degeneracies and Critical Voltage Effects in CBED patterns.
}

\author{
H. Matsuhata,* and J. Gjфnnes
}

* National Institute of Advanced Industrial Science and Technology (AIST), Central 2, 1-1-1, Umezono, Tsukuba, Ibaraki 305-8568, Japan

Centre for Materials Research, University of Oslo, Gaustadalleen 0371 Oslo, Norway

In electron diffraction, the scattering factor is described as below. $\mathrm{f}^{\mathrm{el}}(\mathrm{s})$ and $\mathrm{f}^{\mathrm{x}}(\mathrm{s})$ are the scattering factors for electrons and for x-rays, respectively.

$$
f \text { el }(s)=\left(m e^{2} / 2 h^{2}\right)[Z-f x(s)] / s^{2} .
$$

Where $\mathrm{s}=\sin \theta / \lambda, \theta$ is the Bragg angle, $\lambda$ is the wavelength of an electron , $\mathrm{Z}$ is the atomic number. This indicates scattering factors for low angle reflections obtained by the electron diffraction have advantage in accuracy than the ones obtained from x-ray diffraction.

In the critical voltage effect, one measures the accelerating voltage at which a particular contrast feature in Kikuchi, or CBED pattern appears, along a line or at a point. The effect depends strongly on the structure factors for low angle reflections, and is thus sensitive to the rearrangement of outer electron. The critical voltage effect is explained as an accidental degeneracy of Bloch waves. In diffraction patterns, we can observe various degeneracies of Bloch waves. Not all of degeneracies are the accidental degeneracies which can be used for structure factor estimations. In this report we describe the classifications of various degeneracies of Bloch wave observed in the CBED pattern in $\mathrm{SnO}_{2}, \mathrm{ZnS}$ and $\mathrm{SrTiO}_{3},[1-3]$ and shows some of obtained structure factors of low angle reflections.

Degeneracies observed in CBED patterns are classified as follow.

a) The degeneracies observed at the $3 \mathrm{~m}, 4 \mathrm{~mm}, 6 \mathrm{~mm}$ special points. When the projected point of centre of Ewald sphere on to the zeroth order Laue zone is at $3 \mathrm{~m}, 4 \mathrm{~mm}, 6 \mathrm{~mm}$ point of the zone, we can observe the degeneracies of Bloch waves. However, this is not the accidental degeneracies.

b) When a crystal has a glide-plane or screw axis, we can observe the GM-lines in the CBED discs of forbidden reflections. In the solid-state physics, it is known that the bands degenerate at the Brillouin zone boundary in crystals which belong to the nonsymorphic space group. [4] The B -line of the GM-line corresponds to this degeneracy. This is not the accidental degeneracy.

c) The degeneracies observed on mirror lines. [5] When the projected point of centre of Ewald sphere is on a mirror line of the zeroth order Laue zone, often the degeneracies between mirror and anti-mirror type Bloch waves are observed. These degeneracies are defined as accidental degeneracies in solid state physics textbook. The position of this degeneracy depends on the structure factors as well as the accelerating voltage. Some of the degeneracies move along the mirror-lines by the change of accelerating voltage, and a pair of these degeneracies disappears at the $2 \mathrm{~mm}$ point of the Laue zone. This accelerating voltage is defined to be non-systematic critical voltage.

d) Degeneracy observed on general point caused by the three beam dynamical interaction. [6] This type of degeneracy is considered to be a general case of the accidental degeneracy on mirror-line type $\mathrm{c}$. The degeneracy points move among the general points by the change of the accelerating voltage, and sometimes a pair of the degeneracies points disappear at the two-fold-rotation symmetry 
point on the zeroth order Laue zone.

e) The critical voltage effect of systematic reflections [7], and zone-axis critical voltage effect at $4 \mathrm{~mm}$ and $6 \mathrm{~mm}$ zone axis [9]. This accidental degeneracy is observed at a fixed diffraction condition and at a certain accelerating voltage.

f) Similar phenomenon to critical voltage effect which is observed in non-centrosymmetric crystals. [8] In non-centrosymmetric crystal, often decrease in intensity by the three-beam interaction, similar to the case of critical voltage effect, is observed. In this case the dispersion surfaces branches come closer by the change of accelerating voltage. However, two branches do not touch each other. This type of phenomenon has been used for phase angle estimation of structure factors. [8] The similar phenomenon is known in molecular physics. In an asymmetric molecule energy levels of bonding outer electrons do not degenerate. [10]

By the analysis of systematic critical voltage (e), non -systematic critical voltages (c) and (d), we have estimated the rearrangement of outer -electron in $\mathrm{ZnS}, \mathrm{SnO} 2$ and $\mathrm{SrTiO}$ 3. The example of results for $\mathrm{SrTiO}_{3}$ are summarized in Table 1 . The results clearly show the deviation from the neutral state due to the rearrangement of the outer electrons.

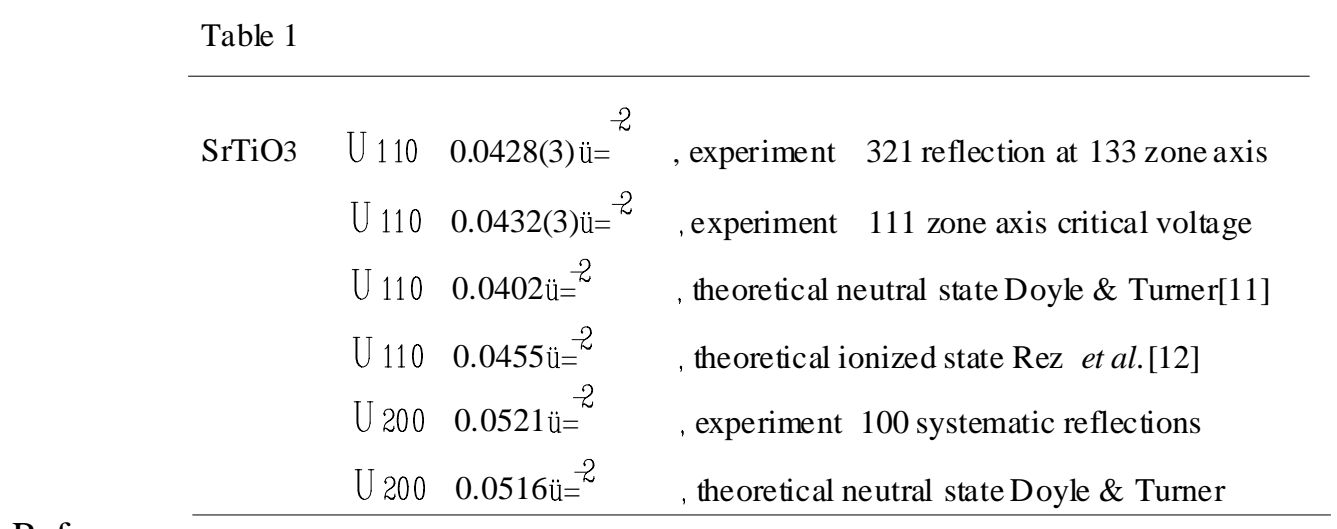

\section{Reference}

[1] H.Matsuhata and J.Gjфnnes, Acta Cryst. A50 (1994) 115.

[2] H.Matsuhata and J.Gj申nnes, Acta Cryst. A52 (1996) 686.

[3] H.Matsuhata and J.Gj申nnes, Proceedings of the Int. Centennial Sympo. on Electron (1997) p470.

[4] G.Burns "Introduction to group theory with applications" New York, Academic press 1977.

[5] J.Taft $\phi$ and J.Gjфnnes, Ultramicroscopy 17 (1985) 445.

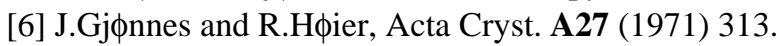

[7] D.Watanabe, R.Uyeda and M.Kogiso Acta Cryst. A24 (1968) 249.

[8] K.Mathinsen and R.H申ier, Acta Cryst. A44 (1988) 558.

[9] M.D.Shannon and J.W.Steeds Philos Mag. 36 (1977) 279.

[10] W.A.Harrison "Electronic structure and properties of solids: the physics of chemical bonds"San Francisco, W.H.Freeman and Company, 1980.

[11] P.A. Doyle and P.S.Turner Acta Cryst A24 (1968) 390.

[12] D.Rez, P.Rez and I.Grant, Acta Cryst A50 (1994) 481. 